\title{
Monitoring FO-PMD and SO-PMD Over Time With Respect to Environmental Conditions
}

\author{
Winston Tumps Ireeta ${ }^{1,2}$, Vitalis Musara ${ }^{1} \&$ Andrew W. R. Leitch ${ }^{1}$ \\ ${ }^{1}$ Optical Fibre Research Unit, Department of Physics, Nelson Mandela Metropolitan University, Port Elizabeth, \\ South Africa \\ ${ }^{2}$ Department of Physics, Makerere University, Kampala, Uganda \\ Correspondence: Winston Tumps Ireeta, Department of Physics, Makerere University, P. O. BOX 7062 , \\ Kampala, Uganda. Tel: 256-752-272-366. E-mail: ireeta@physics.mak.ac.ug
}

Received: January 3, 2014 Accepted: March 6, 2014 Online Published: June 11, 2014

doi:10.5539/apr.v6n4p10

URL: http://dx.doi.org/10.5539/apr.v6n4p10

\begin{abstract}
Environmental conditions affect the birefringence of an optical fibre which in turn leads to fluctuations in its Polarization mode dispersion (PMD). Fluctuations in PMD make compensation for it in optical fibres very complicated. In this paper, fluctuations of first-order polarization mode dispersion (FO-PMD), FO-PMD coefficients and second-order polarization mode dispersion (SO-PMD) are monitored over time and also with respect to the environmental conditions. Long term measurements of FO-PMD have been done before by Mudau (2008) using the Generalized Interferometry Technique (GINTY) only. However, in this current study the FO-PMD coefficients and SO-PMD fluctuations were also monitored in addition to making a comparison between the measurements obtained using the Single-ended Dispersion Analyzer (FTB-5700) and the GINTY. These measurements were made on a $14.8 \mathrm{~km}$ long deployed aerial optical fibre that links St. Albans to Rockland transmission stations in Port Elizabeth, South Africa. The weather information was obtained from the Kestrel 4500 pocket weather tracker that was set up at St. Albans transmission station. So, for good PMD compensation, PMD should be monitored for a very long period of time or use an instrument that can accurately measure it even when the optical fibre is exposed to harsh environmental conditions.
\end{abstract}

Keywords: First-order PMD, Second-order PMD, Generalized Interferometry Technique, Single-ended Dispersion Analyzer

\section{Introduction}

Polarization Mode Dispersion is a form of modal dispersion where two different polarizations of light in a waveguide, which normally travel at the same speed, travel at different speeds due to random imperfections and asymmetries, causing random spreading of optical pulses. The origin of PMD lies in the combined effects of both birefringence and mode coupling. Materials which exhibit birefringence are those where the speed of light is not the same in all directions. Within the amorphous matrix of mechanically unstressed pure glass the $\mathrm{SiO}_{2}$ molecules are randomly distributed. This then makes it a medium which is isotropic in the sense that the physical properties of the glass are uniformly random in all directions.

However, when this glass is exposed to some form of stress, it will exhibit birefringence since this stress will introduce regions of anisotropy within an otherwise amorphous glass matrix. Stress birefringence is due to both intrinsic and extrinsic factors. Intrinsic factors are permanent fibre features that can be accidentally introduced during the manufacturing process for example a non-circular core or non-symmetrical stress fields in the cladding around the core. Extrinsic factors on the other hand are such stresses introduced during cabling and the deployment process.

Environmental factors such as temperature, stress and fibre movement (including wind for an aerial optical fibre) can also lead to changes in the birefringence (Cameron, Chen, Bao, \& Stears, 1998). These effects cause the fibre PMD to vary stochastically in time making PMD particularly difficult to manage. Although amplifiers or other components such as add-drop multiplexers in an optical system may have constant birefringence, variable polarization rotations between them due to the environment cause these components to randomly add to the PMD of the total system (Kogelnik, Jopson, \& Nelson, 2002). 
Within a single mode fibre, there exist random points of perturbation known as mode coupling sites, where energy may couple between the principle modes of adjacent segments. Mode coupling sites are introduced by variations in composition, geometry and strain within the fibre. These could have originated from the fibre preform during the fibre drawing process or even during the cabling process. Stress on the fibre, bends, twists and splices are also other extrinsic sources of mode coupling (Kaminow, 1981).

\section{Measurement Techniques}

The measurement of PMD can be carried out in either the time domain or the frequency domain. The major difference between these two measurement techniques regimes is in the relationship between the coherence time $T_{c}$ of the measurement light and the differential group delay (DGD), $\Delta \tau$ being measured. In the time domain technique, that is when $T_{c}<\Delta \tau$ and in the frequency domain, $T_{c}>\Delta \tau$. Brief discussions of the two measurement techniques follow but the reader is referred to Derickson (1998) and Ireeta, Musara, Wu, and Leitch (2010) for detailed descriptions.

\subsection{Time Domain Measurements}

The time domain measurement techniques determine the PMD by measuring the differential time delay between the slow and fast orthogonal polarization modes of the propagating light pulse. These methods are: time of flight (Williams, 2004), low coherence interferometry (Gisin, Von der Weid, \& Pellaux, 1991; TIA/EIA 455-124-A (FOTP-124A), 2004), polarization-optical time domain reflectometry (Rogers, 1981; Galtarossa \& Palmieri, 2004; Galtarossa, Grosso, Palmieri, \& Schenato, 2008a; Galtarossa, Grosso, Palmieri, \& Schenato, 2008b) and the modulation phase shift method (Williams, 1999).

Other commercially available time domain interferometric techniques which use almost the same principles as the low coherence interferometry are: the FTB-5500 (Traditional Interferometry Technique, TINTY), FTB-5500B (Generalized Interferometry Technique, GINTY) and more recently FTB-5700 (single-ended dispersion analyzer). All these instruments are available from EXFO Electro-Optical Engineering Inc., Quebec City, Canada. These measurement techniques are robust in the field because they facilitate rapid and accurate determination of PMD for even aerial optical fibres which move and vibrate randomly because of the environmental disturbances to which they are exposed.

\subsection{FTB-5500, FTB-5500B and FTB-5700}

The FTB-5500, called "TINTY", is quite similar to the FTB-5500B, called "GINTY". The only difference lies in the inclusion of an algorithm that removes the effects of the central autocorrelation peak (ACP) for the GINTY. This leaves only the cross correlation peaks (CCP) for use to calculate the PMD of a given optical fibre device. It should be noted that the ACP contains no PMD information and it is known to affect the accuracy of measurements of PMD in TINTY (Musara, 2009).

The FTB-5700 is the latest PMD measurement technique to be commercialized by EXFO (Roberge, 2009). Its advantage over GINTY is its ability to make measurements from just one end of the optical fibre link. This makes it a user friendly instrument because it is normally a challenge to have access to both ends of the optical fibre link since they are normally many kilometers apart and also accessibility to both ends at the same time may be quite demanding in terms of human resource. Of course, some researchers have been able to find a solution to this by putting jumpers at one end of the optical fibre link but this could lead to inaccurate results obtained since we are joining two different fibres that have totally different PMDs and properties (though are in the same cable) and the value obtained could be the average of the two (Karlsson, Brentel, \& Andrekson, 2000). It could also be inaccurate false if, for example, one of the fibres has higher PMD compared to the other and yet the final value obtained is the overall of the two or three connected together.

The FTB-5700 is able to measure PMD of aerial optical fibre cables subjected to aeolian-induced vibrations and mechanical perturbations because it has a polarization instability filtering method implemented within it. This is achieved since it uses the state of polarization scrambling analysis method which is completely immune to polarization instabilities when pairs of optical frequency transmission values are simultaneously sampled (Roberge, 2009). In this equipment (FTB-5700), this is done by performing serial measurements on the pair of optical frequencies per measurement point. Hence, executing a series of measurements on the optical transmission pair implies that the state of polarization (SOP) remains stable during the measurement period.

The single-ended dispersion analyzer (FTB-5700) can perform PMD measurements in two regimes, namely: the stable and unstable polarizations. In the stable regime, it uses the serial optical transmission measurement scheme while in the unstable regime a second measurement for the second optical frequency, due to for example wind, is added (Roberge, 2009). 
The experimental setup for the PMD measurements using both the GINTY and the FTB-5700 is shown in Figure 1.

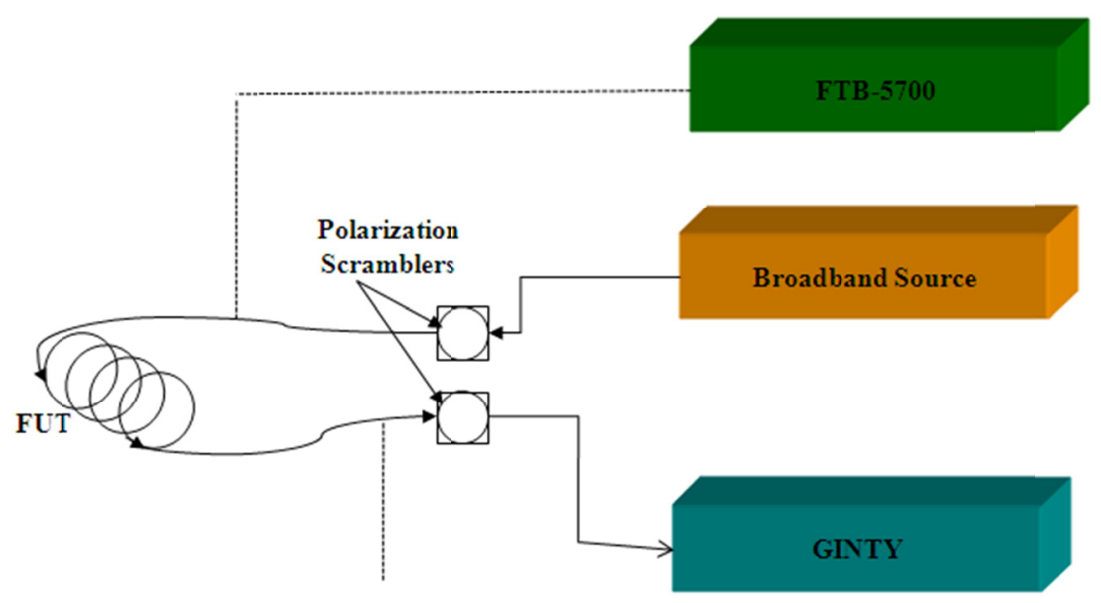

Figure 1. Experimental setup for measurement of FO-PMD, SO-PMD using the FTB 5500B (GINTY) and the FTB-5700

For measurement of the PMDs using the GINTY, the setup consists of a polarized broadband source, FUT (aerial optical fibre under test), polarization scramblers and the GINTY which is composed of the Michelson interferometer, polarization beam splitter (PBS), analyzers and the two detectors. The polarized broadband light is coupled into the FUT and the output light is then launched into the GINTY.

When using the FTB-5700, Single-ended dispersion analyzer, it is connected to the FUT. The other end of the FUT is left open as shown in Figure 1. The collection of the weather data was done every minute so that the FO-PMD and SO-PMD variations are monitored more accurately with time.

\section{Results and Discussions}

\subsection{Fluctuation With Time}
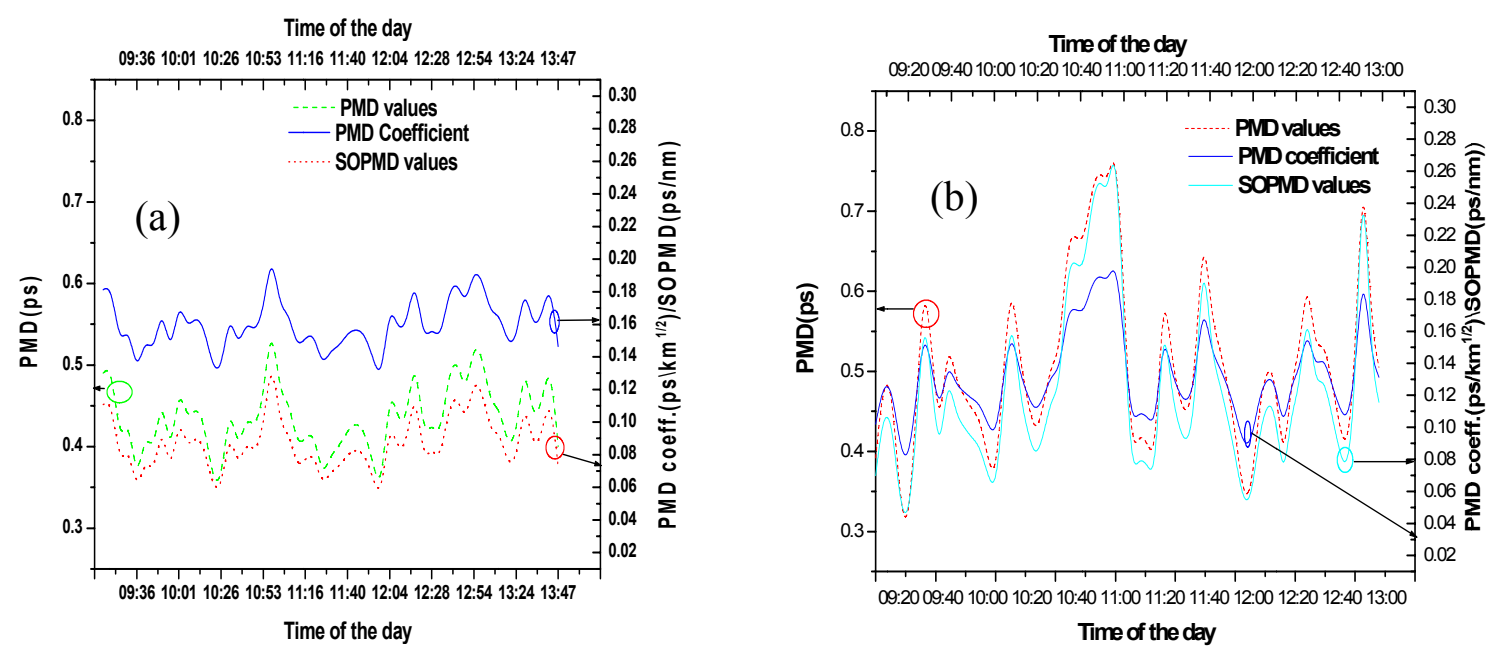

Figure 2. The fluctuation of FO-PMD, FO-PMD coefficients and SO-PMD measured on a deployed aerial optical fibre that links St. Albans and Rockland transmission stations in Port Elizabeth. These measurements were made on 24 August 2010 from 09:00 am to 14:00 pm using (a) FTB-5700, and (b) GINTY 
Figure 2 shows the fluctuations of the FO-PMD, FO-PMD coefficients and SO-PMD with respect to time. These measurements were carried out during the month of August 2010 (spring season) but the results presented here are for 24 August 2010 from 09:00 am to 14:00 pm. During this measurement period the mean temperature was $15.5^{\circ} \mathrm{C}$ and the mean wind speed was $22.7 \mathrm{~km} / \mathrm{h}$. The parameter fluctuations when the FTB-5700 instrument was used are shown in Figure 2(a) while those when the GINTY was used are shown in Figure 2(b). From both these graphs, it is clear that generally the parameter fluctuations for both instruments follow the same trend. In other words, the peaks and troughs for both instruments happen at almost the same time. A case in point is that the maximum FO-PMD, FO-PMD coefficient and SO-PMD values for both instruments are obtained at approximately 10:55 am. Another observation from the graphs is the smaller fluctuation of the FO-PMD values for the FTB-5700 instrument as compared to the GINTY. The former's values fluctuate between $0.35 \mathrm{ps}$ and $0.52 \mathrm{ps}$ yet those for the latter fluctuate between $0.35 \mathrm{ps}$ and $0.78 \mathrm{ps}$.

For this reason, the robustness of the FTB-5700 single-ended dispersion analyzer instrument is tested for its ability to accurately measure PMD of an aerial optical fibre. This is because the PMD of the aerial optical fibre in use is about $0.46 \mathrm{ps}$ so the deviation from it is more pronounced with GINTY compared to the FTB-5700. The same pattern is also observed for the FO-PMD coefficient values as well as the SO-PMD values.

It should be noted that these random fluctuations of these PMD parameters are due to environmental changes especially temperature and wind speed since they are known to change the birefringence and mode coupling of an optical fibre.

\subsection{FO-PMD Fluctuation With Environmental Effects}

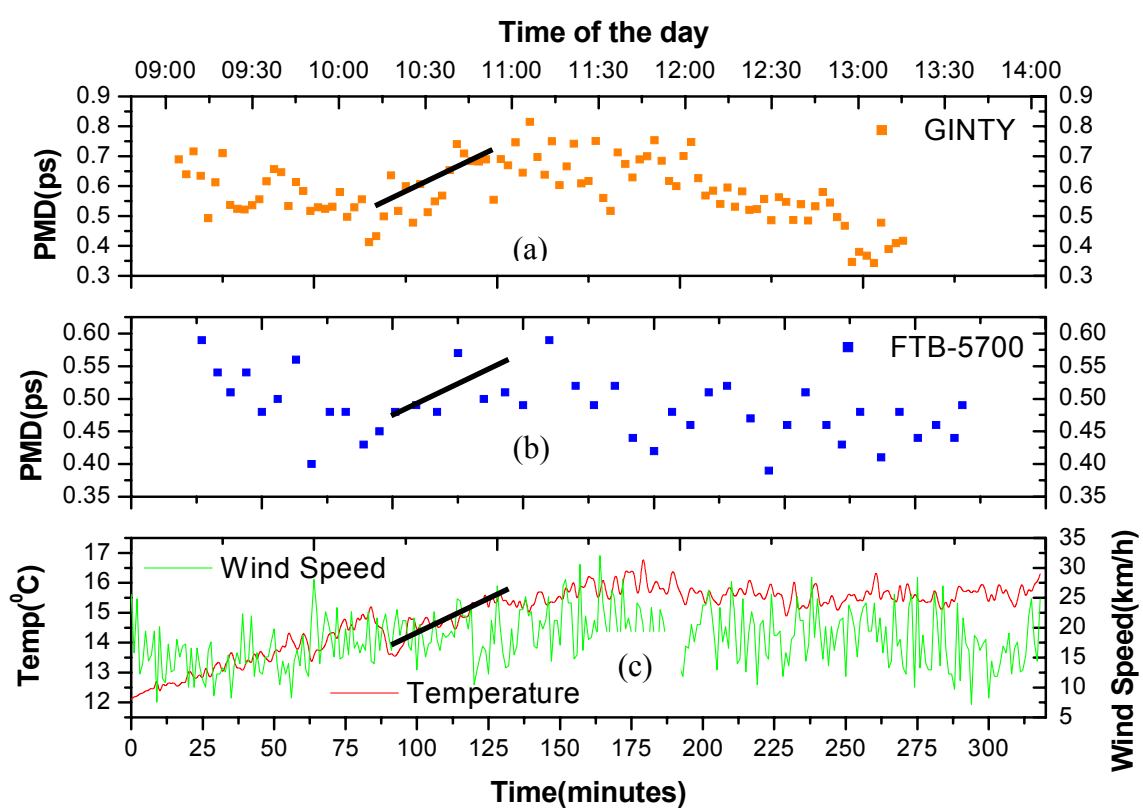

Figure 3. Fluctuation of FO-PMD measured on a deployed aerial optical fibre that links St. Albans and Rockland transmission stations in Port Elizabeth. These measurements were made on 23 August 2010 from 08:45 am to 14:00 pm using (a) GINTY and (b) FTB-5700. (c) Temperature and wind speed as a function of time (the line represents the increase in PMD with temperature)

Figure 3 shows the variations of FO-PMD with respect to time for the GINTY and the FTB-5700 as well as the temperature and wind speed variations. The measurements presented in this section were taken on 23 August 2010 from 08:45 am to 14:00 pm. It should however be noted that more measurements were done during August 2010, though not shown, and they showed the same trend. During this measurement period, the mean temperature was $14.9^{\circ} \mathrm{C}$ with maximum and minimum temperatures of $17.1^{\circ} \mathrm{C}$ and $12.1^{\circ} \mathrm{C}$ respectively. As indicated in Figure 3(c), the temperature fluctuations are small but gradually increased with time until it was approximately constant from 12:00 noon. The wind speeds were however fluctuating randomly with a mean of $18 \mathrm{~km} / \mathrm{h}$, a minimum of $7.2 \mathrm{~km} / \mathrm{h}$ and a maximum of $32 \mathrm{~km} / \mathrm{h}$. This still lies in the vortex-induced oscillations' range. The FO-PMD fluctuations when the GINTY was used are shown in Figure 3(a) while those when the 
FTB-5700 instrument was used are shown in Figure 3(b). Generally, the FO-PMD variations for both instruments are in a random pattern. This is due to the rapid variations in the wind speeds and small variations in the temperature as shown in Figure 3(c). These FO-PMD fluctuations are due to the change in the state of polarization (SOP) of the laser pulse resulting from the combined effect of the change in birefringence and the mode coupling as well as the Berry phase effect on the aerial optical fibre cable. However, a particular trend is observed from Figures 3(a) and 3(b). An increase in FO-PMD is observed starting at 10:15 am to about 11:00 am with a peak achieved for both instruments. A closer look, during this time period, on the temperature graph (Figure 3(c)), a gradual increase in temperature is observed.

This implies that the increase in FO-PMD was due to the increase of the temperature of the aerial optical fibre. However, after 12:00 noon, the temperature variations became quite small. This implies that the random fluctuations of the FO-PMD in both instruments are mainly due to the rapid fluctuations in the wind speeds. This is in agreement with Mudau (2008), Karlsson, Brentel, \& Andrekson (2000) and Zhang, Bao, Yu, \& Chen (2007) who made long-term FO-PMD measurements only that they did not make a comparison of the GINTY with the FTB-5700 during their measurements and also did not show FO-PMD variations for short time (in this particular case a 5.3 hour period is considered).

\section{Conclusion}

Environmental changes, especially temperature and wind speed, change the birefringence and mode coupling of the aerial optical fibre and this in turn leads to the fluctuation in the measured PMDs. It was observed that these parameters varied more when the GINTY was used compared to the FTB-5700 measurement instrument. This confirmed the robustness of the FTB-5700 single-ended dispersion analyzer instrument to accurately measure PMD of an aerial optical fibre.

\section{Acknowledgements}

This work was supported by Telkom SA Ltd, Ingoma Communication Services, Hezeki Contracting, Dartcom, African Laser Centre, National Research Foundation, National Laser Centre, and THRIP.

\section{References}

Cameron, J., Chen, L., Bao, X., \& Stears, J. (1998). Time evolution of polarization mode dispersion in optical fibres. IEEE Photon. Technol. Lett., 10, 1265-1267. http://dx.doi.org/10.1109/68.705611

Cyr, N. (2004). Polarization mode dispersion measurements: Generalization of the interferometric method to any mode coupling regime. IEEE J. Lightwave Technol, 22, 794-805. http://dx.doi.org/10.1109/JLT.2004.824564

Derickson, D. (1998). Fiber Optic Test and Measurement. Prentice Hall. New Jersey.

Galtarossa, A., \& Palmieri, L. (2004). Reflectometric measurements of polarization properties in optical-fiber links. IEEE J. Trans. on Instrumentation and Measurement, 53, 86-94. http://dx.doi.org/10.1109/TIM.2003.821505

Galtarossa, A., Grosso, D., Palmieri, L., \& Schenato, L. (2008a). Reflectometric measurement of birefringence rotation in single mode optical fibers. Opt. Lett., 33, 2284-2286. http://dx.doi.org/10.1364/OL.33.002284

Galtarossa, A., Grosso, D., Palmieri, L., \& Schenato, L. (2008b). Distributed polarization mode dispersion measurement in fiber links by polarization-sensitive reflectometric techniques. IEEE Photon. Technol. Lett., 20, 1944-1946. http://dx.doi.org/10.1109/LPT.2008.2005482

Gisin, N., Von der Weid, J. P., \& Pellaux, J. P. (1991). Polarization mode dispersion of short and long single-mode fibers. IEEE J. Lightwave Technol., 9, 821-827. http://dx.doi.org/10.1109/50.85780

Ireeta, W. T., Musara, V., Wu, L., \& Leitch, A. R. W. (2010). Statistical Characterization of the Output State of Polarization in Deployed Single-Mode Fibres. Opt. Lett., 35, 2049-2051. http://dx.doi.org/10.1364/OL.35.002049

Kaminow, I. P. (1981). Polarization in optical fibres. IEEE J. Quantum Electron. QE-17, 15-22. http://dx.doi.org/10.1109/JQE.1981.1070626

Karlsson, M., Brentel, J., \& Andrekson, P. A. (2000). Long-term measurement of PMD and polarization drift in installed fibers. IEEE J. Lightwave Technol., 18, 941-951. http://dx.doi.org/10.1109/50.850739

Kogelnik, H., Jopson, R. M., \& Nelson, L. E. (2002). Polarization-mode dispersion. In I. Kaminow \& T. Li (Eds.), Systems and Impairments, Optical Fiber Telecommunication IVB (pp. 725-861). New York: Academic Press. 
Mudau, A. E. (2008). Characterization of polarization effects on deployed aerial optical fibre in South Africa. MSc. thesis, Nelson Mandela Metropolitan University (NMMU).

Musara, V. (2009). Polarization mode dispersion emulation and the impact of high first order PMD segments in optical telecommunication systems. PhD thesis, Nelson Mandela Metropolitan University (NMMU).

Roberge, R. (2009). Case study: PMD measurement on aerial fibre under wind-induced oscillations and vibrations. EXFO Electro-Optical Engineering Inc. Technical Note 039.

Rogers, A. J. (1981). Polarization-optical time domain reflectometry: a technique for the measurement of field distributions. Appl. Opt., 20, 1060-1074. http://dx.doi.org/10.1364/AO.20.001060

TIA/EIA 455-124-A (FOTP-124A). (2004). Polarization mode dispersion measurement for single mode optical fibres by interferometry. Telecommunications Industry Association (TIA) Std. Arlington, VA.

Williams, P. A. (1999). Modulation phase-shift measurement of PMD using only four launched polarization states: a new algorithm. Elect. Lett., 35, 1578-1579. http://dx.doi.org/10.1049/el:19991068

Williams, P. A. (2004). PMD measurement techniques and how to avoid pitfalls. J. Opt. Fibre. Comm. Rep., 1, 84-105. http://dx.doi.org/10.1007/s10297-004-0010-4

Zhang, Z., Bao, X., Yu, Q., \& Chen, L. (2007). Time evolution of PMD due to tides and sun radiation on submarine fibers. Opt. Fiber Technol., 13, 62-66. http://dx.doi.org/10.1016/j.yofte.2006.07.003

\section{Copyrights}

Copyright for this article is retained by the author(s), with first publication rights granted to the journal.

This is an open-access article distributed under the terms and conditions of the Creative Commons Attribution license (http://creativecommons.org/licenses/by/3.0/). 